M. Schroeter ${ }^{1,2}$, J. Klein ${ }^{1,2,3}$, F. Erbguth ${ }^{2,4}$, R. Kiefer ${ }^{2,5}$, T. Neumann-Haefelin $^{2,6}$, C. Redecker ${ }^{2,7}$, H. Reese ${ }^{2,8}$, M. Schilling ${ }^{2,9}$, H. Steinmetz ${ }^{2,10}$, G. R. Fink ${ }^{1,2}$

${ }^{1}$ Klinik und Poliklinik für Neurologie, Uniklinik Köln, Köln, Deutschland; ${ }^{2}$ Kommission 1.4 Anhaltszahlen und Qualitätssicherung der Deutschen Gesellschaft für Neurologie (DGN), Berlin, Deutschland; ${ }^{3}$ Seminar Medizinökonomie, Rheinische Fachhochschule Köln, Köln, Deutschland;

${ }^{4}$ Klinik für Neurologie, Klinikum Nürnberg, Nürnberg, Deutschland; ${ }^{5}$ Neurologische Klinik, AGAPLESION Diakonieklinikum Rotenburg gGmbH, Rotenburg/W., Deutschland; ${ }^{6}$ Neurologische Klinik, Klinikum Fulda, Fulda, Deutschland; ${ }^{7}$ Neurologische Klinik, Klinikum Lippe-Lemgo, Lemgo, Deutschland; ${ }^{8}$ Neurologische Klinik, LVR Klinik Bonn, Bonn, Deutschland; ${ }^{9}$ Klinik für Neurologie mit Institut für translationale Neurologie, Universitätsklinikum Münster, Münster, Deutschland; ${ }^{10}$ Klinik für Neurologie, Universitätsklinikum/Goethe-Universität Frankfurt, Frankfurt, Deutschland

\title{
Ergebnisse der 14. Erhebung der Deutschen Gesellschaft für Neurologie zur Struktur der Neurologischen Kliniken mit Akutversorgungsauftrag in Deutschland
}

\section{Einleitung}

Seit 1980 führt die Deutsche Gesellschaft für Neurologie (DGN) alle 2 Jahre eine Umfrage unter deutschen Kliniken mit akutneurologischer Versorgung durch, um Anhaltszahlen zur stationären Versorgung neurologischer Patienten und Patientinnen zu erhalten [1-6]. In Fortschreibung und Weiterentwicklung der Vorumfragen berichten wir hier über die Umfrage, die wir im Jahr 2020 für das Berichtsjahr 2019 durchführten.

Im Vorfeld der Umfrage versandten wir an die Kliniken, die nicht auf die Vorumfrage reagiert hatten, einen Kurzfragebogen, um Optimierungsmöglichkeiten bei der Planung und Gestaltung der Umfrage auszuloten. Auch hierüber berichten wir.

\section{Zusatzmaterial online \\ Zusätzliche Informationen sind in der Online-Version dieses Artikels (https://doi.org/10.1007/s42451-021-00358-y) enthalten. Beitrag und Zusatzmaterial stehen Ihnen auf www.springermedizin.de zur Verfügung. Bitte geben Sie dort den Beitragstitel in die Suche ein, das Zusatzmaterial finden Sie beim Beitrag unter "Ergänzende Inhalte”.}

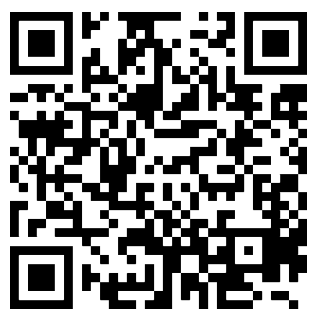

\section{Redaktion}

P. Berlit, Berlin

DGNeurologie 2021 - 4 (5): 332-344

https://doi.org/10.1007/s42451-021-00358-y

Angenommen: 14. Juni 2021

Online publiziert: 19. Juli 2021

(c) Springer Medizin Verlag GmbH, ein Teil von Springer Nature 2021
Die Aussendung erfolgte während der ersten Welle der COVID-Pandemie. Auch wenn uns die zusätzliche Belastung durch die Umfrage bewusst war, hielten wir es für besonders wichtig, die letztmalige Chance zu nutzen, Daten über die Zeit vor der Pandemie zu erheben. Denn seit der Umfrage zum Berichtsjahr 2011 wurde der Fragebogen in großen Teilen konstant gehalten, um Daten vergleichen zu können und so Trends und Entwicklungen anhand der Antworten über die Jahre möglichst valide zu erfassen. Dies ist wiederum Voraussetzung dafür, dass diese Daten als Grundlage strategischer Entscheidungen in den Kliniken genutzt werden können.

Essenzielle Veränderungen des Fragebogens sollten auf aktuelle Fragstellungen eingehen: Im Bereich Infrastruktur wurden die Fragen zu Thrombektomiemöglichkeiten weiter präzisiert, um ein besseres Bild darüber zu bekommen, inwieweit inzwischen ein flächendeckender Zugang zu Thrombektomien in Deutschland erreicht wurde.

Im Bereich Personal interessierte, ob sich der in der letzten Umfrage erhobene dramatische Personalmangel sowohl im pflegerischen als auch im ärztlichen Bereich konsolidiert hat. Dabei wurde erfragt, in welchem Umfang Kliniken gezielt im Ausland rekrutieren und inwieweit Bemühungen unternommen werden, diese Fachkräfte allgemein und fachlich in Deutschland zu integrieren.

Wie in den Vorumfragen war es ein wesentliches Ziel, Benchmarks zu entwickeln, die sich in Bezug zu klinikeigenen Daten setzen lassen, und damit Klinikleitungen Argumente, Kennzahlen und Merkmale zur Beurteilung der Leistungsfähigkeit der eigenen Abteilung zu geben. Dabei war davon auszugehen, dass die DRG-System-immanenten Anreize zu einem weiteren sog. Kellertreppeneffekt mit Fallzahlsteigerung, Verweildauerverkürzung und weiter schleichendem Verfall des durchschnittlichen Fallerlöses/ „casemix“-Index führen [5, 7].

Den teilnehmenden Kliniken wurde zugesichert, dass die Daten anonym bleiben und die Veröffentlichung keine Rückschlüsse auf einen einzelnen Standort erlaubt. Deswegen beschloss die Kommission, auf eine Kartierung von Ergebnissen, z. B. zur Verfügbarkeit der Thrombektomie in Deutschland, zu verzichten. 
Hier steht eine Anzeige.

Springer 


\section{Ergebnisse der 14. Erhebung der Deutschen Gesellschaft für Neurologie zur Struktur der Neurologischen Kliniken mit Akutversorgungsauftrag in Deutschland}

\section{Zusammenfassung}

Die Kommission 1.4 Anhaltszahlen und Qualitätssicherung der Deutschen Gesellschaft für Neurologie führt alle 2 Jahre eine Umfrage unter den an der Akutversorgung neurologischer Patienten teilnehmenden neurologischen Kliniken in Deutschland durch. Anhaltszahlen zur akutneurologischen Versorgung in Deutschland, der Infrastruktur der Krankenhäuser und der neurologischen Fachabteilungen aus dem Jahr 2019 wurden 2020 erfragt und mit den Ergebnissen der Vorumfragen verglichen.

Bei hoher Antwortquote von $67 \%$ gehörte zu den wesentlichen Ergebnissen, dass die Größe der Kliniken und deren Ertragsstärke seit 2015 weitgehend stagnieren. Dies korreliert zeitlich mit dem seit 2015 anhaltenden, die Versorgung einschränkenden Pflegepersonalmangel und dem im Vergleich zu 2017 leicht gemilderten Ärztemangel. Der Ärztemangel konnte durch Rekrutierung von Ärzten aus dem Ausland abgemildert werden. Mindestens einen Arzt mit einem Examen von außerhalb der Europäischen Union beschäftigten $71 \%$ der Kliniken. Nur eine Minderheit bietet spezielle Fortbildungsangebote für aus dem
Ausland rekrutierte Ärzte an. Ambulante Strukturen wurden weiter ausgebaut.

Zur Thrombektomie müssen Patienten von $1 / 3$ der Kliniken verlegt werden und mehr als $50 \mathrm{~km}$ Transportweg überwinden. Im Vergleich zu 2017 erfolgen zumindest zeitweise Thrombektomien in insgesamt deutlich mehr Kliniken. Allerdings führten 48 Kliniken durchschnittlich weniger als 1 Thrombektomie/Woche durch.

Obwohl etwa 1/3 der Krankenhäuser, aus denen Antworten eingingen, sog. Kalkulationshäuser sind, erhielt nur die Hälfte der neurologischen Fachabteilungen an diesen Häusern differenzierte Rückmeldungen zur Kostenstruktur.

Die Anhaltszahlenumfrage zum Jahr 2019 zeigt schlaglichtartig Problemfelder neurologischer Kliniken in Deutschland auf, die aktuell durch die Pandemielage überdeckt sein mögen, aber bereits jetzt als Herausforderungen für die Rückkehr zur Normalität identifiziert werden können.

\section{Methoden}

In Vorbereitung auf die geplante Umfrage wurden im Herbst 2019141 Kliniken angeschrieben, die bei der Vorumfrage nicht geantwortet hatten. Aufgrund der Ergebnisse, insbesondere wegen des Wunsches nach einem kurzen Fragebogen, wurde der Fragebogen überarbeitet. Bei dieser Gelegenheit wurde auch nach der Größe der Kliniken gefragt, um einen Indikator dafür zu gewinnen, ob sich die nicht antwortenden Kliniken systematisch von den antwortenden unterscheiden.

Die eigentliche Umfrage richtete sich an alle bettenführenden neurologischen Kliniken (Hauptabteilungen) in Deutschland, die 2019 vollstationäre Akutbehandlungen nach dem DRG-System abrechneten und an der Notfallversorgung teilnahmen.

Das elektronische Zusatzmaterial zeigt den überarbeiteten Fragebogen zu den Anhaltszahlen des Jahres 2019. Er wurde erstmals im April 2020 an 342 Kliniken ausgesandt. Es folgten im Verlauf 3 weitere Aussendungen per Post. Die zurückgesandten Fragebögen (Eingänge bis zum 01.11.2020) wurden zunächst alle in die Analyse einbezogen. Bei der Datenanalyse wurden jedoch Daten von Kliniken eliminiert, die aufgrund von Strukturbesonderheiten der jeweiligen Klinik zu Extremwerten führten:

- Keine eigenen Betten: $n=1$

- Bettenstruktur dominiert von Rehabilitation (Phase B/C/D) $n=7$

- Bettenstruktur dominiert von Geriatrie $n=1$

Die Ausfüllhinweise zum Fragebogen betonten ausdrücklich die Möglichkeit, Fragen offen zu lassen, wenn keine sicheren Angaben gemacht werden konnten; damit weicht bei spezifischen Antworten die Gesamtzahl der Antworten von der o.g. Zahl $219 \mathrm{ab}$.

Die Auswertung und grafische Aufarbeitung erfolgten weitgehend mit Excel (Microsoft, Seattle, WA, USA). Die Ergebnis- se haben den Charakter einer Stichprobe, deren Normalverteilung nicht sicher beurteilbar war. Entsprechend beschränken wir uns im Folgenden auf deskriptive Maße der Statistik mit der Angabe von z. B. Median, oberem bzw. unterem Quartil sowie der Spannbreite (Range) und verzichteten auf Angaben des arithmetischen Mittelwertes und der Standardabweichung. Dass jedoch die durchschnittliche Bettenzahl bei der Vorumfrage und bei der jetzigen Umfrage bei 56 Betten lag, ist ein starker Hinweis darauf, dass die Umfrageergebnisse eine repräsentative Stichprobe darstellten.

\section{Ergebnisse}

Von 228 Kliniken gingen Antworten ein. Damit lag die Teilnahmequote mit $67 \%$ über der der Vorumfrage (61\%). Es gingen die Daten von 18 Uniklinika ein, die damit gut repräsentiert waren (64\% aller Universitätsklinika), ähnlich wie in den Vorumfragen.

Unverändert lag zu 2015 und 2017 die Gesamtbettenzahl aller Kliniken bei einem aktuellen Median von 56 Betten, mit Grenzen des unteren (25\%-) Quartils bei 40 Betten (d.h., $25 \%$ der Kliniken hatten höchstens 40 Betten) und des oberen (75\%-) Quartils bei 73 Betten (d.h., 25\% der Kliniken hatten mehr als 73 Betten). Dasselbe galt für die Größe der Stroke-Units, die ähnlich groß angegeben wurden, wie 2 Jahre zuvor: Median 9 Betten (2017: 8), Quartilsgrenzen bei 6 bzw. 12 Betten.

148/219 der Kliniken gaben keine neurologisch betriebenen Intensivbetten an, also immerhin $68 \% .18 \%$ der Kliniken verfügten über 6 oder mehr eigene Intensivbetten; eine Bettenzahl, die eine Größe darstellt, die als eigenständige Intensivstation mit vertretbarem Aufwand betreibbar erscheint. Die Auswirkungen dieses Umstandes auf die Weiterbildungsbefugnis werden weiter unten diskutiert. 


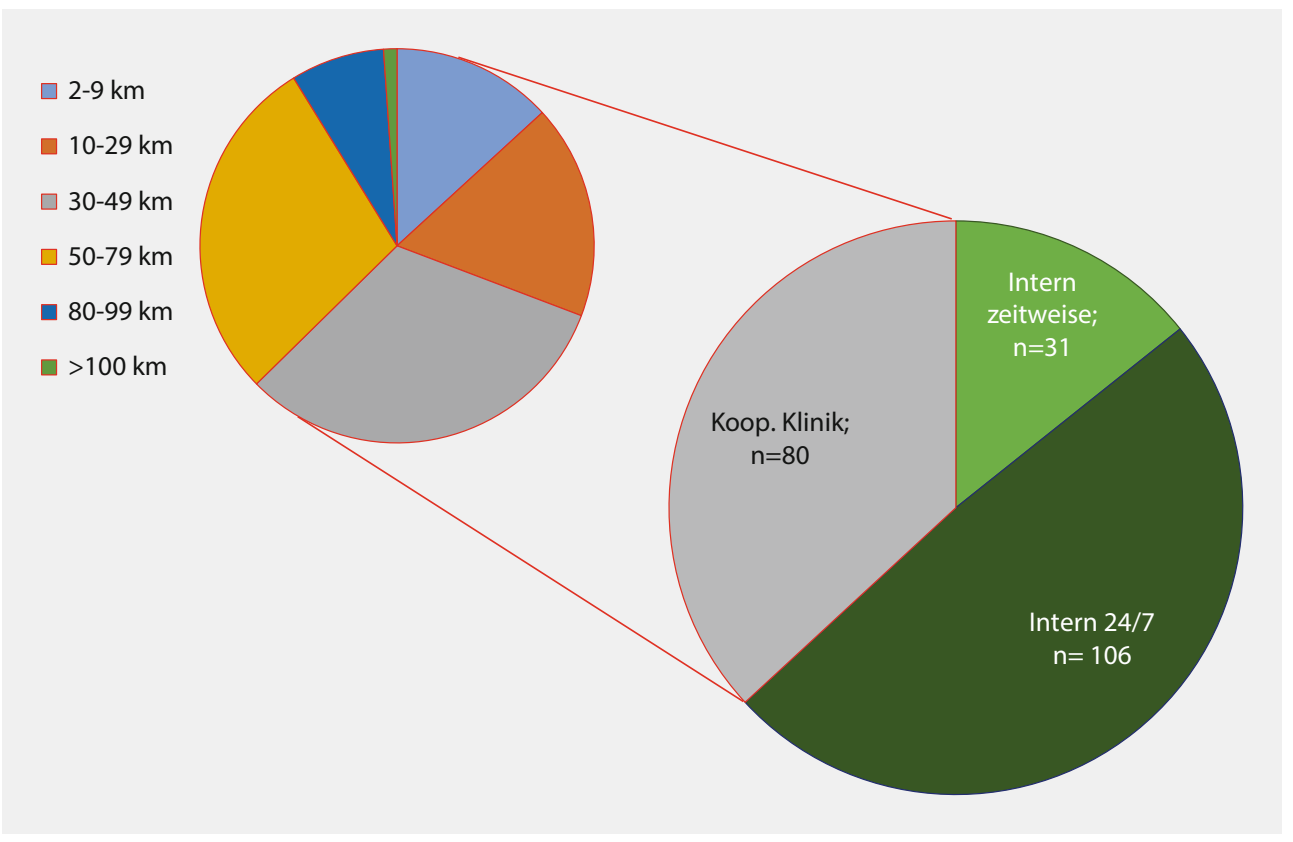

\begin{abstract}
Abb. 1 Verfügbarkeit der Thrombektomie (TE). Während eine Mehrzahl von Kliniken angibt, TE zumindest zeitweise intern anbieten zu können (großes Kreisdiagramm, grüne Segmente), müssen etwa $40 \%$ der Kliniken Patienten zur TE überweisen (graues Segment), etwa 1/3 von ihnen immer noch über Entfernungen von mehr als $50 \mathrm{~km}$ (kleines Kreisdiagramm). Koop. kooperierende
\end{abstract}

Es wurde auch wieder nach Betten „in anderen Bereichen“ gefragt: 24 (2017: 21) Kliniken gaben geriatrische Betten an, 5 (10) Kliniken Betten im Bereich Palliativmedizin.

Neben der vor- und nachstationären Behandlung im engen zeitlichen Zusammenhang mit einer stationären Behandlung hielten 136/219 (62\%) der neurologischen Kliniken mindestens eine ambulante Versorgungsstruktur vor: 35 eine Poliklinik (16\%), 110 eine Ermächtigung (50\%), 50 (22\%) eine $\$ 116$ b-Ambulanz, 73 (33\%) eine Praxis im Krankenhaus oder ein MVZ. Während die anderen ambulanten Versorgungsformen konstant waren, stieg die Zahl der Kliniken mit Praxis am Krankenhaus und/oder MVZ um 10\% binnen 2 Jahren.

Bei der zentralen Notfallversorgung verfestigte sich die Einbindung der Neurologie in interdisziplinäre Notaufnahmestationen. 182/210 Kliniken bezeichneten sich als fallführend in den Notaufnahmestationen (87\%, unverändert zu 2017; 2015: $82 \%)$.

Je mehr die Neurologie zum Notfallfach wird, desto wichtiger wird es, die dafür notwendige diagnostische Infrastruktur rund um die Uhr verfügbar zu haben.

Die Notfallversorgung in den neurologischen Kliniken ist geprägt von der Schlaganfallversorgung, und diese wiederum durch die schnellstmögliche Identifikation von Patientinnen und Patienten, die einer Lysetherapie oder Thrombektomie (TE) zugeführt werden müssen. Diese Entwicklung wird nochmals verstärkt durch jüngere Studien, die anhand von komplexen Bildgebungsinformationen Schlaganfallerkrankte definierten, die bei unklarem Zeitfenster oder jenseits von 4,5-6h von einer Lyse oder TE profitieren können [8-10]. Damit ist es mit hoher Evidenz für alle Kliniken, die mit einer Stroke-Unit an der akuten Schlaganfallversorgung teilnehmen, notwendig, nicht nur rund um die Uhr eine MR- oder CT-Angiographie der hirnversorgenden Gefäße durchführen zu können, sondern auch zusätzlich Bildgebungsmodalitäten zur Verfügung zu haben, die potenziell rettbares Gewebe identifizieren (z. B.
FLAIR-Diffusions-Mismatch, Perfusionsbildgebung in CT und MRT).

Während alle Kliniken einen Zugriff auf CT und CT-Angiographie angaben, hatten nur 159/216 Kliniken (74\%) rund um die Uhr Zugriff auf ein MRT, damit immerhin jedoch $10 \%$ mehr als vor 2 Jahren. 64/217 (29\%) Kliniken führten telemedizinische Befundungen durch, identisch zur Umfrage 2017, jedoch mit steigendem Prozentsatz zu den Umfragen davor.

Die TE hat einen festen Platz in der Akutversorgung des ischämischen Schlaganfalls erlangt. Während in 2015 nur 56\% der Kliniken Zugang zur TE hatten, gaben jetzt wie 2017 alle Kliniken mit Stroke-Units an, Zugang zur TE zu haben (Abb. 1). Beachtliche 49\% der Kliniken (2017:44\%) gaben an, die TE im Haus rund um die Uhr durchzuführen, weitere $14 \%$ können zumindest zeitweise TE leisten. 37\% waren bei TE-Indikationen auf eine Verlegung in kooperierende Kliniken angewiesen. Wir fragten wie in der Vorumfrage, welche Entfernung zum TE-Zentrum zurückgelegt werden muss, wenn die TE nicht rund um die Uhr vor Ort angeboten wird. Hierbei war eine zweigeteilte Entwicklung zu verzeichnen: Die Zahl der Kliniken, aus denen für die TE eine Entfernung von 10-29km zurückzulegen war, nahm deutlich ab (2017: 31 Kliniken, 2019: 16 Kliniken), während für weitere Entfernungen die Zahlen unverändert blieben. Bei $1 / 3$ der Kliniken sind über $50 \mathrm{~km}$ zum TE-Zentrum zurückzulegen (Abb. 1). Auch wenn wir mit Rücksicht auf die zugesicherte Anonymität der Daten auf eine Veröffentlichung einer entsprechenden Landkarte für Deutschland verzichten, so ist doch qualitativ festzustellen, dass die Versorgungsdichte in Deutschland für die TE heterogen bleibt. Die Etablierung neuer Zentren erfolgte offenbar kleinräumig in Regionen, in denen bereits entsprechende Angebote vorhanden waren, während in der Fläche, in der große Entfernungen zur TE zu überwinden sind, keine zusätzlichen TE-Zentren entstanden.

Für die Zertifizierung als überregionale Stroke-Unit wird eine Mindestzahl von 35 TE pro Jahr gefordert, eine Zahl von 50 


\begin{tabular}{|c|c|c|c|c|c|c|c|}
\hline & $\begin{array}{l}\text { Median } \\
2011\end{array}$ & Median & $\begin{array}{l}\text { Median } \\
2015\end{array}$ & $\begin{array}{l}\text { Median } \\
2017\end{array}$ & Median & $\begin{array}{l}\text { Unteres } \\
\text { Quartil }\end{array}$ & $\begin{array}{l}\text { Oberes } \\
\text { Quartil }\end{array}$ \\
\hline Vollstationäre Fälle $(n)$ & 2383 & 2408 & 2525 & 2600 & 2572 & 1901 & 3308 \\
\hline Davon: Stroke-Unit & 729 & 750 & 826 & 804 & 830 & 651 & 1200 \\
\hline Notfallkontakte & 1800 & 2109 & 1954 & 2264 & 2196 & 1095 & 4047 \\
\hline "Casemix" & 2443 & 2526 & 2713 & 2614 & 2976 & 1720 & 3601 \\
\hline "Casemix"-Index & 1,04 & 1,03 & 1,08 & 1,01 & 1,01 & 0,91 & 1,15 \\
\hline Durchschnittliche Verweildauer (Tage) & 6,8 & 6,5 & 5,4 & 6,04 & 5,9 & 5,2 & 6,6 \\
\hline
\end{tabular}

empfohlen. Für neurovaskuläre Netzwerke beträgt die Mindestzahl 75 TE pro Jahr im koordinierenden Zentrum. Für die Ausweisung eines neurovaskulären Zentrums (Spitzenzentrum Schlaganfallmedizin) nach den Vorgaben des gemeinsamen Bundesausschusses werden 100 TE pro Jahr gefordert [11, 12]. Im Mittel wurde in unserer Umfrage die Durchführung von 82 TE pro Jahr angegeben, mit Quartilen bei 27 bzw. bei 118 TE. 38 Kliniken führen mehr als 100 TE durch, 50 Kliniken mehr als 75; 72 Kliniken mehr als 50/Jahr, 46 jedoch weniger und damit auch weniger als $1 \mathrm{TE}$ pro Woche. Dabei gab es große Unterschiede hinsichtlich der Zuweisungszahlen aus externen Kliniken: Unter den Kliniken, die mehr als 100 TE durchführten, lag der Prozentsatz extern zugewiesener TE-PatientInnen zwischen 5 und $80 \%$.

Fallzahl, „casemix“ und „casemix“-Index (CMI) sind 3 globale Parameter, mit denen die Leistungsfähigkeit einer Klinik eingeschätzt wird und mit denen Kliniken untereinander verglichen werden. Auf sie beziehen sich auch gängige Effizienzkennzahlen (vgl. Abb. 2). Tab. 1 stellt die Mediane den Werten von 2011-2019 gegenüber und gibt für das aktuelle Berichtsjahr zusätzlich die Grenzen für das untere und obere Quartil an.

Im Median wurden knapp 2600 Fälle, davon 830 auf der Stroke-Unit, behandelt. Die Verweildauer betrug 5,9 Tage im Median, der mediane "casemix“ von 2880 entsprach 2019 bei einem Bundesbasisfallwert von $3544,97 €$ einem Erlös von 10.209 Mio. €. Dieser deutliche Erlöszuwachs im Vergleich zu 2017 (+14\%) war teilweise durch den Anstieg des Bundesbasisfallwertes um 4,8\% und teilweise durch den Anstieg der Summe der CM-Punkte (+9,2\%) erklärt, während sich zumindest in dieser globalen Betrachtung die Fallschwere laut DRG Katalog nicht änderte: Der CMI blieb bei 1,01 im Median.

Insgesamt lassen die Basiszahlen (Tab. 1) einen Expansionstrend von 2011-2015, gefolgt von einer Konsolidierungsphase 2015-2019 erkennen. Die Fallzahlen stagnierten im Median.

Als Effizienzmaße wurde eine Reihe von einfachen Quotienten gebildet. Aus 2019 ist dies auch im Vergleich zu den Vorjahren letztmalig ohne Einschränkungen möglich, die Ausgliederung der Pflegebudgets wird solche Betrachtungen (Abb. 2) in Zukunft erschweren.

Fallzahl und „casemix“ sind einfache Kennzahlen, die den Leistungsumfang einer Klinik beschreiben; die Abb. 2a, b lassen die heterogene Größe neurologischer Kliniken in Deutschland erkennen. Fallzahl pro Vollkraft (VK) und „casemix“/ Vollkraft sind Benchmarks für die Personalbemessung einer Abteilung. Beide Werte fallen gegenüber der Vorerhebung deut- lich ab von ca. 160 bzw. 190 auf 128 im Mittelwert. Es wird also offenbar mehr Personal eingesetzt, um die Leistungen zu erbringen. Hinter diesen Zahlen können sich vielfältige Entwicklungen verbergen (s. Diskussion). Dabei ist bei einem durchschnittlichen CMI von 1 (vgl. Tab. 1) zu erwarten, dass beide Zahlen im Durchschnitt nominell sehr ähnlich sind. Weichen bei einer individuellen Klinik die Zahlen voneinander derart ab, dass die FZ/VK kleiner ist als der „casemix“/VK, so spricht dies für ein in Bezug auf den finanziellen Erlös günstiges Fallportfolio der Klinik (und umgekehrt).

Ein zweites wichtiges Zahlenpaar im Benchmarking sind „casemix“/Belegungstag und CMI-Inkrement/Belegungstag (Abb. 2e, f). Im Mittel stieg dieser Wert 2019 im Vergleich zu 2017 von 0,17 CM-Punkten/Tag auf 0,18 CM-Punkte/Tag an. Berücksichtigt man gleichzeitig den Anstieg des Bundesbasisfallwertes von 3376,11 auf $3544,97 €$, so ergibt sich hieraus ein Tageserlös von 639,52€ gegenüber 607,03€ in 2017 $(+32,49 €$ oder $+5,35 \%)$. Diese Zahlen geben also einen durchschnittlichen Tageserlös wieder und machen DRG-Erlöse einer Tagespauschale grob vergleichbar. Dabei wird impliziert, dass jeder Verweildauertag innerhalb einer DRG gleich viel Erlös bringt. Wenn dies im Mittel der Fall wäre, so sollte das durchschnittliche Ansteigen des CMI pro Belegungstag bei einem CMI von 1 dazu führen, dass die beiden Größen CM Punkte/Tag und CMI Inkrement/Tag nominell identisch sind. Dies ist hier tatsächlich der Fall (0,1839 bzw. 0,1840).

Jenseits dieser Benchmarks zu Erlösen mit Bezug zu Personaleinsatz und Verweildauer ist es ein zentrales Ziel des Krankenhauscontrollings, Kostenstrukturen zu erfassen. Hierbei ist für alle Kalkulationshäuser die vom InEK vorgegebene Matrix für eine Kostenträgerrechnung bindend. 69 (32\%) Kliniken gehörten zu den sog. Kalkulationshäusern, 117 nicht. Bemerkenswerterweise war es 31 Kliniken unbekannt, ob sie Kalkulationshäuser sind. In den teilnehmenden $32 \%$ der Kliniken, die Kalkulationshäuser sind, wurden $36 \%$ der hier erfassten Fallzahlen behandelt. Aus den 69 Kalkulationshäusern erhielten allerdings nur 39 Kliniken differenzierte Rückmeldungen zur Kostenträgerrechnung; immerhin 24 erhielten hierzu keine Informationen, und 6 Klinikleitungen war dies völlig unbekannt.

Das Diagnosenportfolio der Kliniken bietet einen weiteren Ansatz für die vergleichende Analyse neurologischer Kliniken. Wir fragten nach den Top-5-DRG in der Dreistellersystematik (z. B. B70). Leider war die Antwortqualität hier sehr heterogen: Diese Angaben fehlten nicht nur bei einem Teil der Kliniken, es wurden häufig DRG-Fallzahlen in der Vierstellersystematik angegeben (z.B. B70B), einige gaben auch Fallzahlen bezogen 
Hier steht eine Anzeige.

Springer 

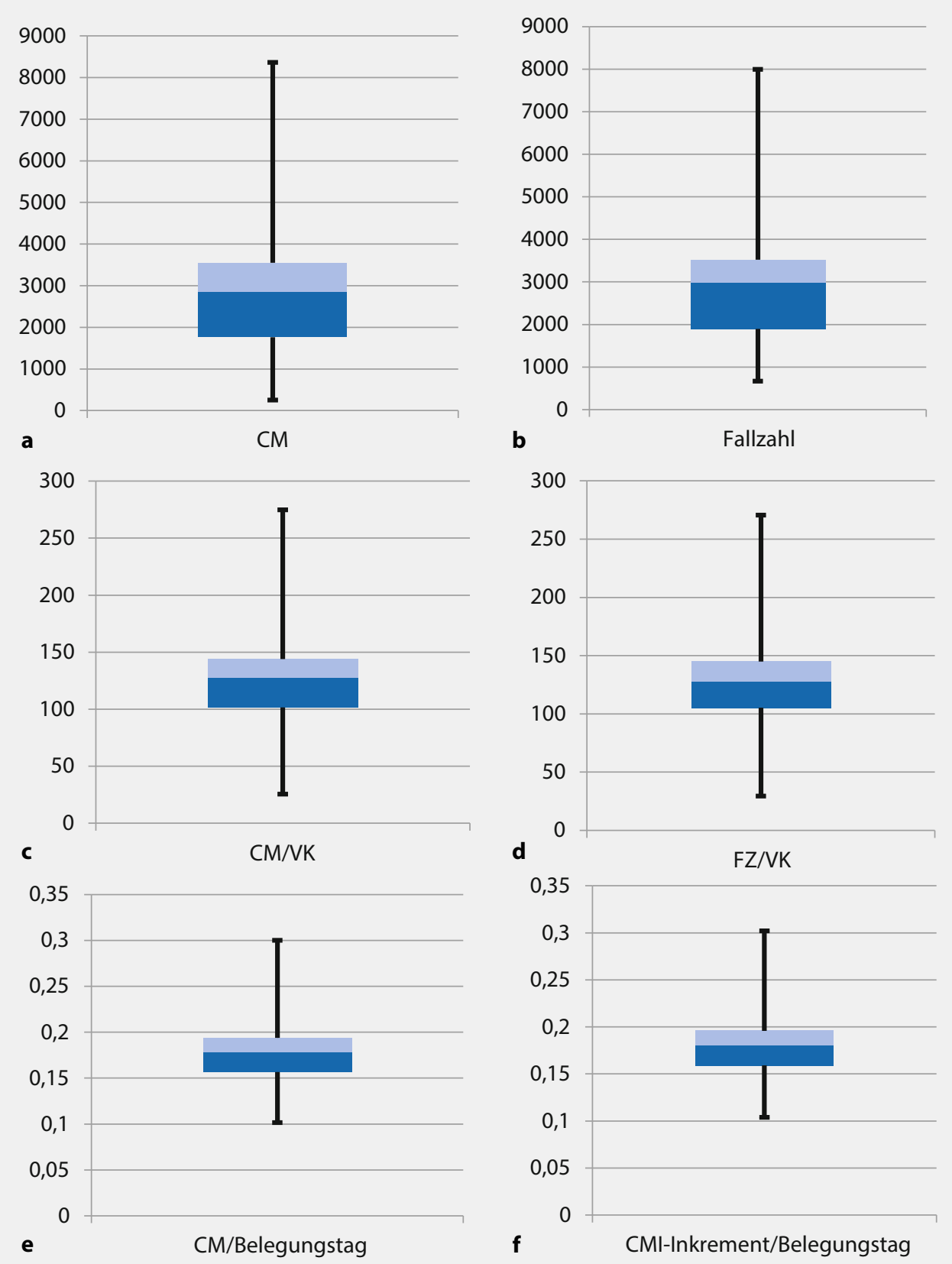

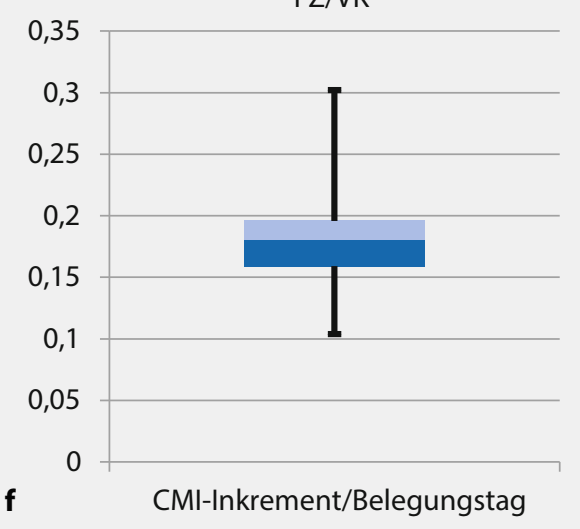

Abb. 2 Benchmarks. Box-und Whisker-Plots von "casemix" (a; $C M)$ und Fallzahlen (b) sowie 4 Effektivitätsmaßen: „casemix“ pro Vollkraft $(V K ; \mathrm{c})$, Fallzahl $(F Z)$ pro Vollkraft (d), "casemix" pro Belegungstag $€$ und Inkrement des "casemix"-Index (CMI) pro Belegungstag (f). Die untere Box gibt das 2. Quartil, die obere das 3. Quartil, den Mittelwert abgrenzend wieder, die Whisker die Wertespanne. Die Grafik ermöglicht das Abtragen klinikindividueller Benchmarks, zur Interpretation: s. Diskussion auf ICD-Diagnosen in Drei- und Vierstellern an (z. B. I63 bzw. I63.4). Wir entschieden uns deshalb gegen eine weitere diesbezügliche Auswertung.

Ausgehend von der Situation, dass praktisch alle neurologischen Kliniken in Deutschland eine Stroke-Unit vorhalten, boten die Fallzahlen für die Komplexbehandlung des akuten Schlaganfalls (OPS 8-981) einen guten Anhalt, wie dominant die Schlaganfallversorgung im Leistungsportfolio einer Klinik ist. Überraschend homogen ergab sich bei den meisten Kliniken ein Anteil der Schlaganfallpatienten und -patientinnen mit Komplexbehandlung von 28\% (Mittelwert; Quartile 22 bzw. $32 \%$; Abb. 3). Die Hauptdiagnose Schlaganfall machte in den meisten Kliniken etwa 1/3 der Behandlungsfälle aus. Klinik- modelle, die monothematisch auf eine Schlaganfallversorgung setzten, waren in der Versorgung nicht persistent.

Die Vorumfrage zu den Anhaltszahlen 2017 hatte einen dramatischen Anstieg des Personalmangels ergeben: Erstmals hatten mehr als die Hälfte der Kliniken angegeben, die Versorgung zeitweise wegen eines Mangels an Ärzten und/oder Pflegepersonal eingeschränkt haben zu müssen. 2019 betraf dies erneut die Mehrzahl der Kliniken (54\%). Dabei waren die Entwicklungen im pflegerischen und ärztlichen Bereich gegenläufig: Während der Ärztemangel sich leicht entspannte, nahm der Pflegemangel trotz bereits zuvor hohem Niveau noch einmal leicht weiter zu. Die Abb. 4 veranschaulicht die Entwicklung von 2013-2019.

Nicht nur der nominelle Mangel an Mitarbeitenden kompliziert die Personalplanung an den Kliniken, auch die Perso- 


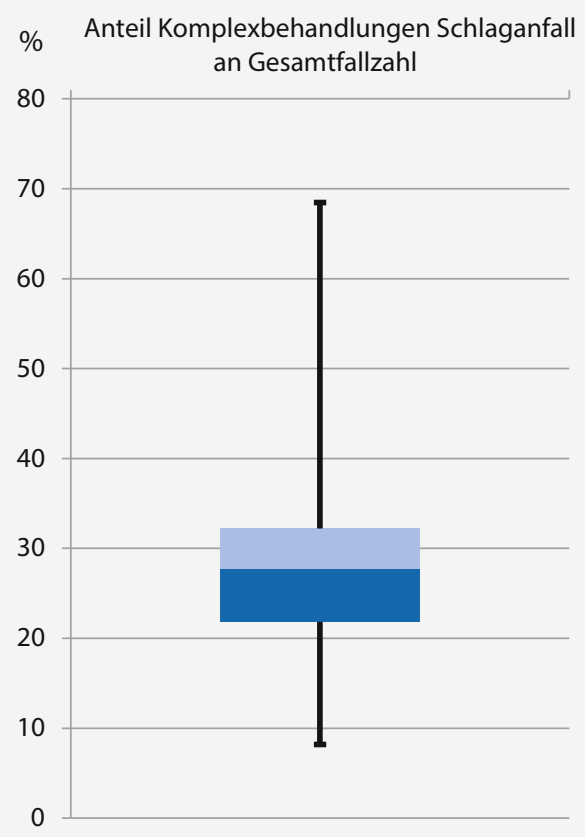

a

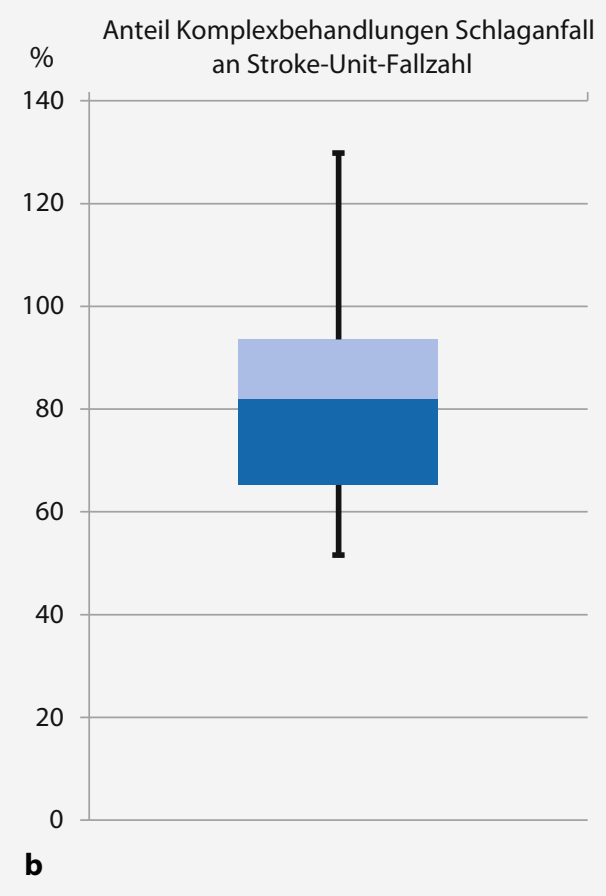

Abb. 3 Bedeutung der Schlaganfallkomplexbehandlung für das Leistungsportfolio der Kliniken. Komplexbehandlungen Schlaganfall haben recht homogen einen Anteil von 25-30\% an den Fallzahlen der Kliniken (a). Dagegen ist der Anteil von Komplexbehandlungen an den Fallzahlen auf Stroke-Units bemerkenswert heterogen (b). Zur Systematik der Box-und WhiskerPlots s. Abb. 2

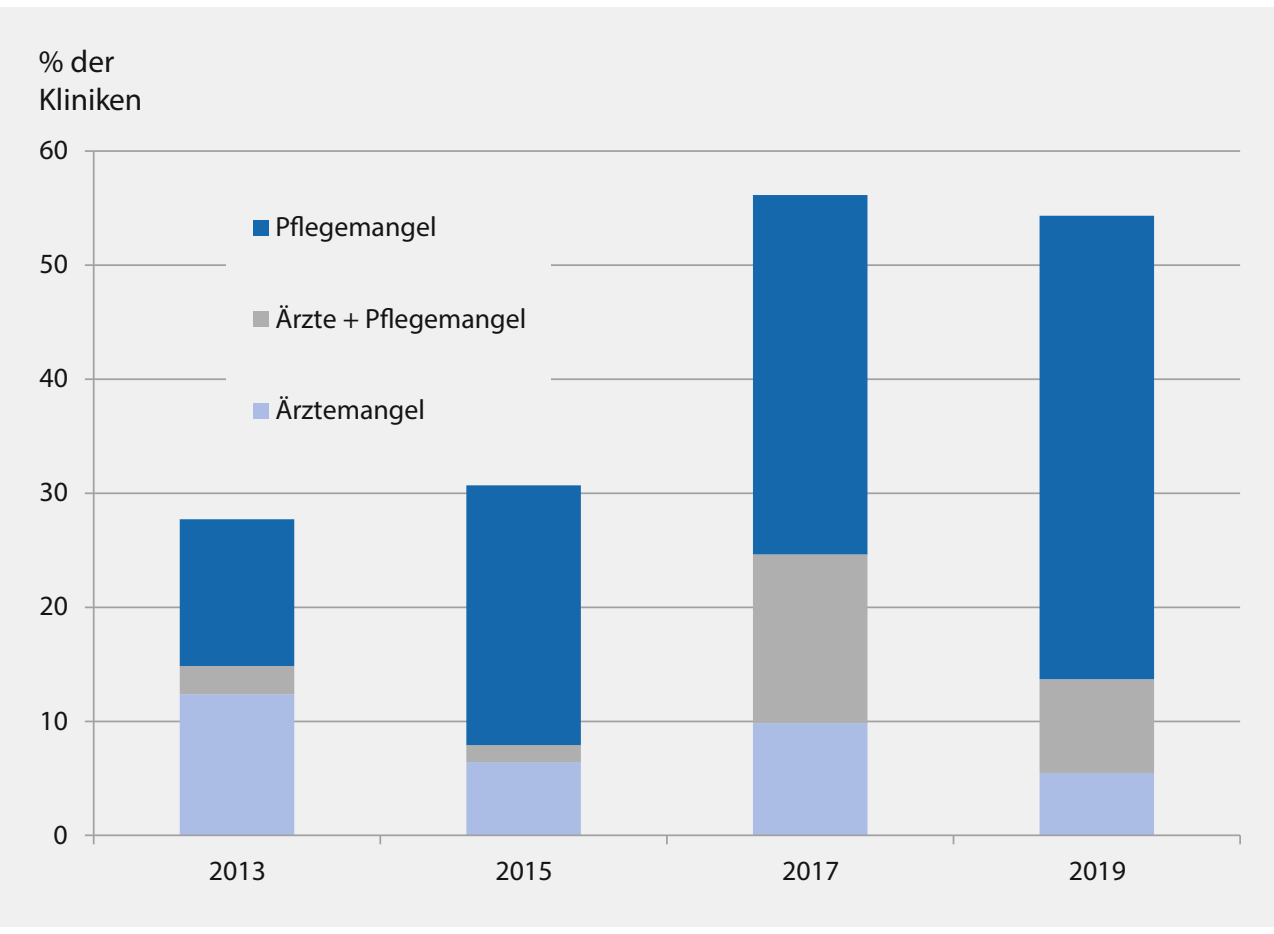

Abb. 4 Ärzte- und Pflegemangel. Die Grafik zeigt die Anzahl der Kliniken (in \% von allen antwortenden Kliniken) von 2013-2019, die aufgrund Pflegemangels (dunkelblau), Ärztemangels (hellblau) oder beidem (grau) ihre Versorgung einschränken mussten

nalstruktur hat sich in den letzten 10-15 Jahren deutlich geändert (Abb. 5): individuelle Zeitmodelle, kurzfristige („Kind krank“) oder monatelange (z. B. Beschäftigungsverbote im Mutterschutz, Elternzeiten) Ausfälle ohne oder mit nur kurzer Vorlaufzeit erschweren die Planung. Die Rekrutierung von ausländischem ärztlichem Personal kann nominellen Mangel kompensieren, die Mitarbeitenden kämpfen jedoch mit der neuen Sprache, einem neuen, komplizierten deutschen Gesundheits- system und neuen medizinischen Standards, die die Arbeitsleistung über Monate schmälern. Einige Zahlen, die diese Herausforderungen als Mittelwert pro Klinik angeben, fasst Abb. 6 zusammen.

Trotz der vielen zu integrierenden ausländischen Ärzte und Ärztinnen waren die spezifischen Fortbildungsangebote für diese Gruppe spärlich. Während 31 Kliniken aktives Recruiting von ärztlichem Personal im Ausland angaben, hatten nur 


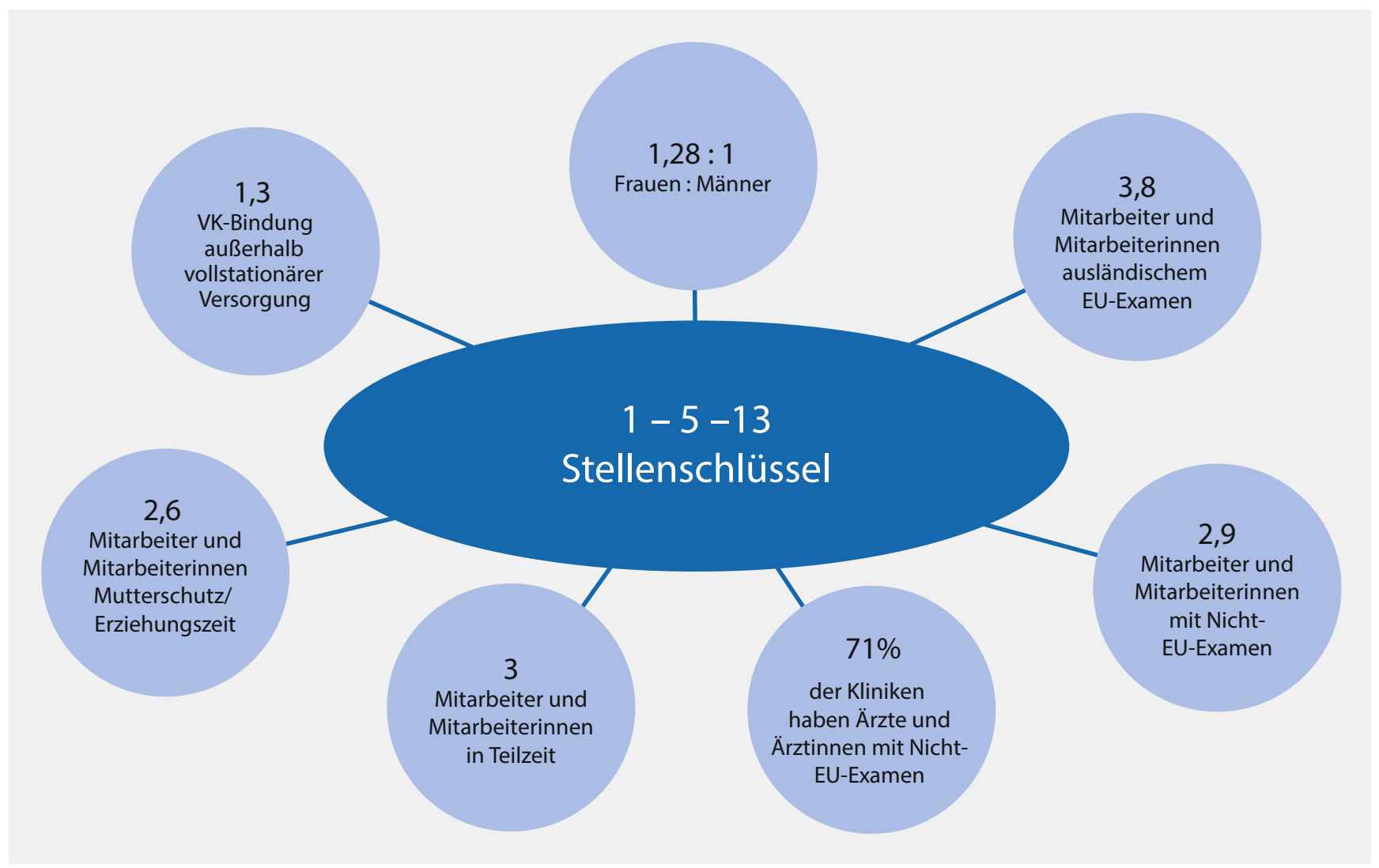

Abb. 5 ÄrztInnen: „human ressource“, wichtige Kennzahlen zur ärztlichen Ausstattung der Kliniken, EU Europäische Union, VK Vollkraft

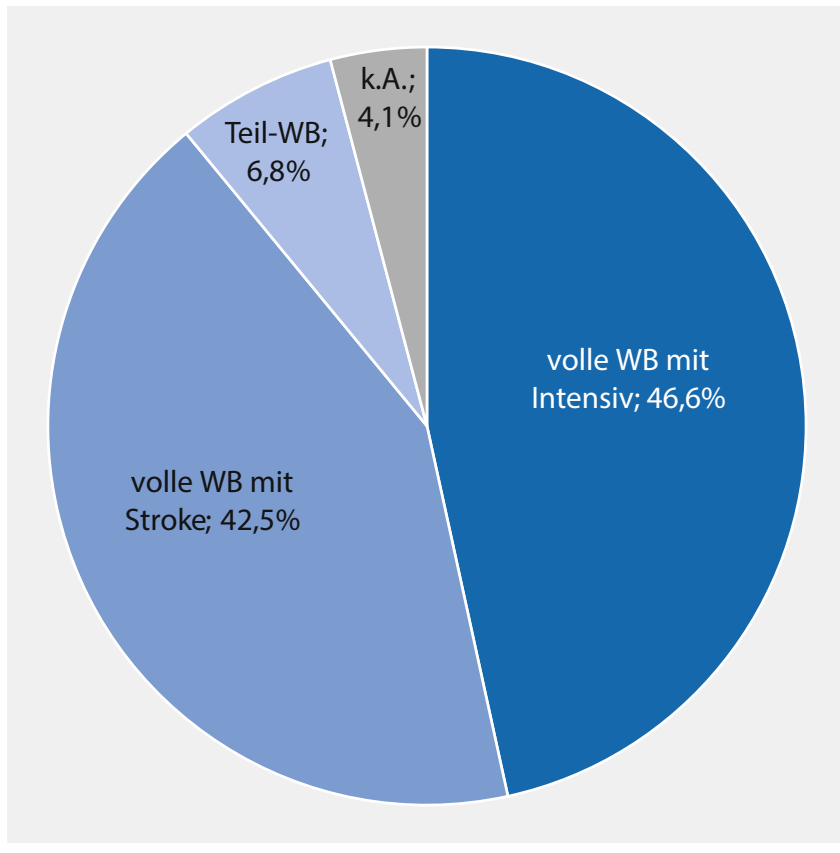

Abb. 6 Weiterbildungsermächtigung. Die Großzahl der Kliniken besitzt eine volle Weiterbildungsermächtigung. Von diesen stellen die Hälfte die Weiterbildungsinhalte zur Intensiv- und Akutmedizin über die Stroke-Unit dar. WB Weiterbildung
11 (35\%) davon spezifische Fortbildungsangebote für solche mit Migrationshintergrund (hier wurde nicht einmal nach spezifisch neurologischen Fortbildungsangeboten gefragt). Aus der Gesamtheit von 212 Kliniken, die diese Frage beantworteten, hatten $13 \%$ ein solches Fortbildungsangebot, wobei $71 \%$ der Kliniken mindestens einen Arzt/eine Ärztin mit Nicht-EUExamen beschäftigten.

Eine Teilweiterbildungsermächtigung lag nur bei 15/219 Kliniken vor. Der Großteil der Kliniken gab an, eine Weiterbildungsermächtigung für die vollen 48 Monate Neurologie zu besitzen. Dabei stellten knapp die Hälfte hiervon den 6-monatigen Abschnitt zur intensiv- und akutmedizinischen Ausbildung über die Stroke-Unit her.

\section{Diskussion}

Hiermit legen wir die 14. Auflage der Anhaltszahlenumfrage der DGN vor. Die Kliniken nehmen an dieser fragebogenbasierten Umfrage freiwillig und unentgeltlich teil, müssen aber für das Ausfüllen des Fragebogens einen gewissen Zeitaufwand investieren. Umso mehr freuen wir uns über die rege Teilnahme mit einer Antwortquote von 67\%. Im Vorfeld der aktuellen Umfrage hatten wir unter den bei der Vorumfrage nicht antwortenden Kliniken erfragt, welche Faktoren denn die Teilnahme verhindert hatten. Hierbei wurde als wesentlicher Faktor Zeit benannt. Dies war Anlass, den Fragebogen weiterhin knapp zu halten, was notwendigerweise dazu führt, dass einige Themenbereiche nur kursorisch erfragt werden können. Als Indikatorgröße erfragten wir bei dieser Vorumfrage die Bet- 
tenzahl der Klinik. Dass unter diesen zuvor nicht antwortenden Kliniken ebenfalls eine durchschnittliche Bettenzahl von 56 Betten zu erfragen war, sehen wir als Indikator dafür an, dass sowohl die aktuelle als auch die vorherigen Anhaltszahlenumfragen repräsentative Stichproben für die neurologischen Akutkliniken in Deutschland darstellen. Als positiven Hinweis auf die Validität der Daten werten wir auch die Konstanz der erhobenen Werte.

Die Aussendung dieses Fragebogens fiel in eine seit dem 2. Weltkrieg nicht gekannte Krisensituation deutscher Krankenhäuser infolge der SARS-CoV-2-Pandemie. Ein Verschieben der Umfrage um 1 Jahr mit dann Erhebung der Daten aus 2020 erschien jedoch nicht sinnvoll: Jede Dynamik der Zahlen wäre als pandemiebedingt zu interpretieren gewesen. Die Zahlen aus 2019 zeigen jedoch, welche Problemfelder bereits vor der Pandemie bestanden hatten und in 2020 möglicherweise durch Corona akzentuiert wurden. Die Anhaltszahlen konkretisieren somit Zielvorstellungen und Herausforderungen für die nächsten Monate bei der Rückkehr zu einer (neuen?) Normalität. Eine verlängerte Rücklauflatenz der Fragbögen nahmen wir dafür in Kauf - immerhin über 6 Monate von der ersten Aussendung bis zur letzten Frist am 01.11.2020.

Für die stationären Basiskennzahlen (Tab. 1) lässt der Vergleich zu den Vorumfragen 2011-2017 [1-6] 2 Phasen erkennen: 2011-2015 war eine Expansion stationärer Leistungen zu erkennen. Seit 2015 stagnieren diese Zahlen. Ursächlich ist dabei der dramatisch zunehmende Personalmangel nach 2015, insbesondere im Pflegebereich, anzunehmen. Bei den absoluten berichteten Zahlen ist zu berücksichtigen, dass wir nach Einschränkungen der bestehenden Versorgung durch Ärzte- oder Pflegemangel fragten. Ein noch kritischerer Einfluss des Personalmangels muss auf mögliche Pläne eines strategisch-expansiven Wachstums einer Fachabteilung und die hierfür nötigen Investitionen angenommen werden. Es ist $\mathrm{zu}$ erwarten, dass durch die Einführung und mit Sanktionen durchgesetzten Pflegepersonaluntergrenzen für neurologische Stationen diese Mangelentwicklung weiter verschärft wird [13, 14].

Die Mangelsituation in der stationären Versorgung setzt einen zusätzlichen Impuls in der ohnehin fortschreitenden Ambulantisierung des Faches. Hier zeigt sich gegenüber der Vorumfrage ein fortschreitender Trend zur Ansiedlung von medizinischen Versorgungszentren oder Praxen am Krankenhaus. Ein neurologisches MVZ oder eine Praxis funktionieren häufig in enger personeller Verzahnung mit der Klinik mit dem immanenten Risiko, dass sich Zusatzaufgaben nicht in einer adäquaten Ausweitung des Personaltableaus niederschlagen. Dabei birgt die Auslagerung von radiologischen Leistungen in ein MVZ oder eine Praxis die Gefahr, dass die neurologische Klinik einerseits an diesen Anbieter gebunden ist, andererseits dessen Preise akzeptieren muss. Über den Preis kommt es dann - auch bei prinzipieller Verfügbarkeit rund um die Uhr - zu einer Verknappung dieser Ressource.

Neben der infrastrukturellen Herausforderung, komplexe Schlaganfallbildgebung rund um die Uhr für rekanalisierende Therapien im sich erweiternden Zeitfenster anzubieten, bedarf es hierfür auch einer vertieften akuten (neuro-)radiologischen Befundungskompetenz. Die teleradiologische Befundung steht dem prinzipiell nicht entgegen. Fällt die Präsenzpflicht des Radiologen, so muss umso mehr aus Sicht der neurologischen
Kliniken eine neuroradiologisch sichere Indikationsstellung und Befundung für die Akutsituation rund um die Uhr gesichert sein.

Die im Dezember 2014 veröffentlichte MR CLEAN-Studie läutete nicht weniger als ein neues Zeitalter in der Schlaganfallversorgung ein [15]. Während 2015 Thrombektomien (TE) nur regional begrenzt verfügbar waren, bieten inzwischen alle Stroke-Units den direkten oder mittelbaren Zugang zur TE an [16]. Auch wenn zwischen 2017 und 2019 viele Krankenhäuser dazu kamen, die selbst zumindest zeitweise die TE anbieten, so kann weiterhin nur eingeschränkt von einer flächendeckenden Versorgung gesprochen werden. TE-Raten weisen erhebliche regionale Unterschiede auf [17]. In unverändertem Umfang müssen aus gut $1 / 3$ der Stroke-Units, die nicht selber thrombektomieren, über $50 \mathrm{~km}$ zum Weitertransport zurückgelegt werden. Hier erscheint das Qualitätsziel von max. 60-90 min von der verlegenden Klinik bis zum Beginn der Intervention („door-to-groin time“) gefährdet. Dagegen scheinen in den Ballungsräumen zahlreiche TE-Zentren hinzugekommen zu sein, welche die hier schon vorher unkritischen Transportzeiten verkürzen, aber auch eine Konkurrenzsituation herstellen und dem Qualitätsziel von Mindestmengen entgegenstehen. Es ist vorgesehen, eine Mindestzahl von TE bei der Zertifizierung von überregionalen Stroke-Units ab dem 01.01.2022 zu fordern. Siedelte man zusätzliche Stroke-Units mit TE-Möglichkeit in der Fläche an, wäre ein Zielkonflikt zwischen kurzen Transportzeiten und angestrebten Mindestmengen zu befürchten. In einem solchen ländlichen Setting ständen bei niedriger Gesamtfallzahl und kleiner Abteilungsgröße sowie nicht zu erreichenden Mindestfallzahlen hohe Vorhaltungskosten für das Aufrechterhalten der Therapiemöglichkeit gegenüber. Hier bedürfte es also eines politischen Willens und Sondervergütungen, um eine flächendeckende TE-Versorgung zu ermöglichen.

In den Ballungsräumen hingegen zeigt sich der Bedarf nach einer Konsolidierung des (Über-)Angebots von Krankenhausleistungen, der auch als probater Ausweg aus der Personalmangelsituation angesehen wird [18]. Auch hier ist eine politische Willensbildung zu fordern, um eine sog. Bereinigung des Marktes zugunsten einer wirtschaftlich und personell auskömmlichen Situation der verbleibenden Einrichtungen zu erreichen.

Benchmarks für die Ertragsstärke einer Klinik sind Fallzahl, "casemix" und CMI, die in Tab. 1 wiedergegeben werden, aber auch die Quotienten, die in Abb. 2 als Box- und Whisker-Plots grafisch dargestellt sind. Hier bietet es sich an, klinikindividuelle Werte grafisch abzutragen und daraus einen Eindruck zu gewinnen, wo man steht. Eine zusätzliche Information lässt sich aus dem Vergleich der beiden Werte in der 2. und 3. Reihe ziehen:

Im DRG-System kommt es keineswegs zu einem gleichmäßigen, tagesgleich harmonischen Anstieg des Erlöses. Ist der Wert Fallzahl/VK kleiner als der Wert für „casemix“/VK, so kann angenommen werden, dass ein finanziell überdurchschnittlich ertragseffektiver Einsatz des Personals erfolgt. Liegt das CMI-Inkrement (3. Reihe) einer individuellen Klinik über dem CM/Tag-Wert, so kann angenommen werden, dass für das DRG-Portfolio der Klinik eine überdurchschnittlich erlöseffektive Verweildauergestaltung erreicht wurde. Aussagekräftiger werden diese Betrachtungen, wenn sie nicht nur auf Klinik- 
ebene, sondern für spezifische Fallgruppen oder DRG durchgeführt werden.

Während solche Benchmarks zur Erlösstärke (Abb. 2) allgemeine Akzeptanz erfahren, sind differenzierte Betrachtungen $\mathrm{zu}$ Erlösen und Kosten offenbar wenig verbreitet. Selbst in dem Drittel der Kliniken, die zu den Kalkulationshäusern gehören, wurde diese dort notwendigerweise erhobene Kostenträgerrechnung nicht an die Abteilungsleitenden weitergegeben. Dabei könnte gerade in der Kommunikation von medizinischem Controlling und Abteilungsleitung aus der Diskussion von Kostenstrukturen ein kostenbewussteres Wirtschaften im Alltag erreicht werden. Andererseits könnten Lücken und Fehler der Kostenträgerrechnung aufgedeckt werden, was Systemrelevanz im DRG-System besitzt, da nicht erfasste oder zugeordnete Kosten in den Kalkulationshäusern die der entsprechenden DRG zugebilligte Fallschwere und damit die Erlöse in den Folgejahren mindern.

Wir gehen davon aus, dass der eklatante Pflegepersonalmangel ein wesentlicher Faktor für die Stagnation der zuvor expansiven Entwicklung der deutschen Krankenhausneurologie ist, verstärkt durch die Vorgaben der Pflegepersonaluntergrenzen (vgl. o.). Dabei verursachen Gehaltssteigerungen gemäß Tarifvertrag, aber auch darüber hinausgehende Zulagen und Vergünstigungen stark ansteigende Kosten, insbesondere auch bei der Beschäftigung von Honorarkräften. Die Ausgrenzung der Pflegepersonalkosten aus den DRG-Budgets verspricht zwar die kostenneutrale Abdeckung dieser Kosten für die Pflege, gleichzeitig ist dies Gegenstand von Verhandlungen mit den Kostenträgern mit entsprechenden wirtschaftlichen Risiken. Auch ist klar, dass Mehrkosten für Honorarkräfte oder ungelernte Hilfskräfte zur Entlastung der Pflegenden auf Station keineswegs abgedeckt werden und auf Kosten der Abteilungen durchschlagen.

Auch das ärztliche Personal ist von einem absoluten und relativen Mangel betroffen. Während zum Jahr 2007 in der damaligen Umfrage ein Stellenschlüssel von 1-3-8,5 im Median angegeben wurde, betrug dieser jetzt 1-5-13 bei ähnlicher Bettenzahl und durchschnittlich 2025 Fällen im Jahr [1].

Vorgaben des Tarifvertrages im Sinne einer Begrenzung von Bereitschafts- und Wochenenddiensten tragen dazu bei, dass der Personalschlüssel aufgestockt werden muss [19].

Ein erheblicher Anteil der Kliniken rekrutiert aktiv Ärzte aus dem Ausland, oft aus einem nicht EU-Gesundheitssystem ohne Kenntnis der komplexen deutschen Gegebenheiten. Defizite in den Sprach- und Fachkenntnissen stellen sich oft erst nach Arbeitsbeginn heraus. Allerdings zeigt die Umfrage auch ein großes Defizit von spezifischen Fortbildungsmaßnahmen für diese rekrutierten Ärzte, um sie schneller und besser zu integrieren.

Insgesamt kann diese Umfrage sowohl auf der Planungsebene der Krankenhäuser als auch der neurologischen Fachabteilungen wertvolle Informationen zu Entwicklungen und Problemen geben. Auch wenn diese in den letzten Monaten durch die Pandemie verdrängt oder überdeckt waren, so werden die skizzierten Probleme unsere Rückkehr zur Normalität mitbestimmen $[20,21]$.
Literatur

1. Wallesch CW, Brand T, Engelhardt A et al (2008) Ergebnisse der 8. Erhebung zur Struktur der neurologischen Kliniken der Akutversorgung in Deutschland. Aktuelle Neurol 35:359-369

2. Wallesch CW, Fink GR, Engelhardt A et al (2011) Ergebnisse der 9. Erhebung zur Struktur der neurologischen Kliniken der Akutversorgung in Deutschland. Aktuelle Neurol 38:1-11

3. SchroeterM, Engelhardt A, Erbguth FJ et al (2013) Ergebnisse der 10. Erhebung zur Struktur der neurologischen Kliniken der Akutversorgung in Deutschland. Aktuelle Neurol 40:1-10

4. Schroeter M, Lüßem B, Engelhardt A et al (2015) Ergebnisse der 11. Erhebung der Deutschen Gesellschaft für Neurologie zur Struktur der neurologischen Kliniken der Akutversorgung in Deutschland. Aktuelle Neurol 42(02):72-79

5. Schroeter M, Erbguth FJ, Kiefer R et al (2017) Ergebnisse der 12. Erhebung der Deutschen Gesellschaft für Neurologie zur Struktur der neurologischen Kliniken mit Akutversorgungsauftrag in Deutschland. Aktuelle Neurol 44:1-10

6. Schroeter M, Rezazadegan K, Erbguth FJ et al (2019) Struktur der neurologischen Kliniken in Deutschland: Ergebnisse der 13. Erhebung der Deutschen Gesellschaft für Neurologie. DGNeurologie. https://doi.org/10.1007/s42451-019-0078-y

7. Simon M (2013) Das deutsche DRG-System: Grundsätzliche Konstruktionsfehler. Dtsch Arzteb/ 110(39):A-1782/B-1572/C-1548

8. EXTEND-IA Investigators, Campbell BC, Mitchell PJ, Kleinig TJ et al (2015) MRIguided thrombolysis for stroke with unknown time of onset. N Engl J Med 372(11):1009-1018

9. DEFUSE 3 Investigators, Albers GW, Marks MP, Kemp S et al (2018) Thrombectomy for Stroke at 6 to 16 Hours with Selection by Perfusion Imaging. N Engl J Med 378(8):708-718

10. Thomalla G, Simonsen CZ, Boutitie F et al (2018) WAKE-UP investigators. MRIguided thrombolysis for stroke with unknown time of onset. N Engl J Med 379(7):611-622

11. Antrag auf Zertifizierung eines neurovaskulären Netzwerkes. https://www.dgnr. org/de-DE/185/nvn-antragsunterlagen. Zugegriffen: 20. März 2021

12. Hecken J Beschluss des Gemeinsamen Bundesausschusses über eine Änderung der Regelungen zur Konkretisierung der besonderen Aufgaben von Zentren und Schwerpunkten gemäß § 136c Absatz 5 SGB.www.g-ba.de/downloads/62-4922328/Z-R_2020-11-20_iK-2020-12-18.pdf.Zugegriffen: 20. März 2021 13. Bundesgesetzblatt Jahrgang 2018 Teil1 Nr. 45: Gesezu zur Stärkung des Pflegpersonals. https://Www.bgbl.de/xaver/bgbl/start.xav? startbk=Bundesanzeiger_BGBI\&start=\%2F\%2F\%2A\%5B\%40attr_id $=$ \%27bgbl118s2394.pdf\%27\%5D\#_bgbl_\%2F\%2F*\%5B\%40attr_id\%3D \%27bgbl118s2394.pdf\%27\%5D_1615030735403.Zugegriffen: 20. März 2021

14. Bundesgesetzblatt Jahrgang 2020 Teil Nr. 51: Verordnung zur Festlegung von Pflegepersonaluntergrenzen in pflegsensitiven Bereichen in Krankenhäusern für das Jahr 2021. https://www.gkv-spitzenverband.de/media/dokumente/ krankenversicherung_1/krankenhaeuser/pflegepersonaluntergrenzen/kh_ ppug2021/PpUGV_BGBI_09.11.2020.pdf.ZZugegriffen: 20. März 2021

15. MR CLEAN Investigators, Berkhemer OA, Fransen PS, Beumer Det al (2015) A randomized trial of intraarterial treatment for acute ischemic stroke. N Engl J Med 372(1):11-20

16. Krogias C, Bartig D, Kitzrow M et al (2017) Verfügbarkeit der mechanischen Thrombektomie bei akutem Hirninfarkt. Nervenarzt 88:1177-1185

17. Krogias C, Weber R, Richter D, Bartig D, Eyding J (2020) Bundesweite Versorgungsrealität von Patienten mit akutem Hirninfarkt in Deutschland. Update der regionalisierten Analyse zur Anwendung rekanalisierender Therapieverfahren und einer Schlaganfallkomplexbehandlung. Nervenarzt 91:908-919

18. PD - Berater der öffentlichen Hand GmbH Gutachten - Kurzfassung. Krankenhauslandschaft NRW. Berlin 2019. https://broschuerenservice.mags.nrw/ files/download/pdf/gutachten-krankenhauslandschaft-nrw-kurzfassung-pdf_ von_gutachten-krankenhauslandschaft-nordrhein-westfalen-kurzfassung_vom_ mags_3138.pdf.Zugegriffen:20. März 2021 
Hier steht eine Anzeige.

Springer 
19. Marburger Bund Services: Tarifverträge. marburger-bund.de/bundesverband/ tarifvertraege. Zugegriffen: 20. März 2021

20. Kuhlen R, Winklmair C, Schmithausen D et al (2020) Effekte von COVID-19Pandemie und Lockdown auf die Versorgung von Krankenhauspatienten. Dtsch Arztebl Int 117:488-489

21. Finkenzeller KKranke Krankenhäuser. https://www.zeit.de/wirtschaft unternehmen/2020-08/krankenhaeuser-kliniken-coronavirus-behandlunginsolvenzen-hygieneregeln-pandemie?utm_referrer=https\%3A\%2F\%2Fwww. google.com\%2F. Zugegriffen: 20. März 2021

\section{Korrespondenzadresse}

\section{Prof. Dr. M. Schroeter}

Klinik und Poliklinik für Neurologie

Uniklinik Köln

50924 Köln, Deutschland

michael.schroeter@uk-koeln.de

\section{Einhaltung ethischer Richtlinien}

Interessenkonflikt. M. Schroeter, J. Klein, F. Erbguth, R. Kiefer, T. NeumannHaefelin, C. Redecker, H. Reese, M. Schilling, H. Steinmetz und G.R. Fink geben an, dass kein Interessenkonflikt besteht.

Für diesen Beitrag wurden von den Autoren keine Studien an Menschen oder Tieren durchgeführt. Für die aufgeführten Studien gelten die jeweils dort angegebenen ethischen Richtlinien.

Danksagung. Wir bedanken uns bei den teilnehmenden Kliniken für die Beantwortung der Fragebögen und Frau Magdalena Gier, Köln, für die administrative Unterstützung. Teile dieser Veröffentlichung sind Teil der Masterthesis von Frau Julia Klein am Seminar Medizinökonomie, Rheinische Fachhochschule Köln.
Friedhelm C. Schmitt, Hermann Stefan, Martin Holtkamp (Hrsg.)

\section{Epileptische Anfälle und Epilepsien im} Erwachsenenalter

Heidelberg: Springer-Verlag 2020, (ISBN: 978-3-662-59197-0), 99,99 EUR

Als ich meine neurologische Weiterbildung an der Universitätsklinik Heidelberg begann, gab es noch kaum apparative Methoden in der Neurologie. Was aber trotz fehlender zerebraler Bildgebung schon zum Einsatz kam, war das EEG. Und ich hatte das Vergnügen diese Technik bei Walter Christian zu lernen, der 1957 gemeinsam mit Dieter Janz das Krankheitsbild des Impulsiv Petit mal beschrieben hatte. Ein Video-EEG gab es natürlich noch nicht, aber die Patienten und Patientinnen waren in großen Sälen untergebracht. Der Schreibtisch für Ärztinnen/Ärzte und Pflegende stand mitten in diesen Sälen und wir konnten sämtliche Formen epileptischer Anfälle quasi vom Schreibtisch aus beobachten und beschreiben.

Seither hat sich nicht nur in der Neurologie insgesamt, sondern ganz besonders in der Epileptologie sehr viel getan: mehrfach wurden die Phänomenologie von Anfällen und deren Klassifikation neu erarbeitet und überarbeitet, die Ätiologie zahlreicher Epilepsien konnte geklärt werden, und die Möglichkeiten der Behandlung haben sich vervielfacht. Dem trägt das von F.C. Schmitt, H. Stefan und M. Holtkamp herausgegebene Buch Rechnung, in dem auf nahezu 800 Seiten das aktuelle Wissen zu epileptischen Anfällen und Epilepsien im Erwachsenenalter zusammengetragen wird. Basierend auf der Terminologie der Klassifikation von 2017 werden nach Darstellung der Grundlagen die Anfallstypen, Epilepsiesyndrome und Ätiologien ausführlich dargestellt. Der Abschnitt über die Diagnostik umfasst neben Labor, EEG und Video-EEG-Monitoring die Möglichkeiten der Quellenlokalisation, Magnetenzephalographie, nuklearmedizinische und funktionelle MRT-Untersuchungen, genetische Diagnostik und stellt histopathologische Befunde vor.

Bei der Pharmakotherapie werden Mono- und Polytherapien ausführlich dargestellt. Eigene Kapitel widmen sich dem Konzept der Pharmakoresistenz und den Möglichkeiten der Beendigung einer medikamentösen Behandlung. Ausführlich werden die verschiedenen Verfahren der Epilepsiechirurgie und Neuromodulation erläutert sowie komplementäre Therapien wie ketogene Diät oder Psychoedukation.

Für den praktischen Alltag in Klinik und Praxis ist die ausgewogene Darstellung legaler und psychosozialer Aspekte ausgesprochen hilfreich. Besonders zu erwähnen sind dabei die Auflistungen von Hilfsangeboten für Patienten und Patientinnen wie Apps oder auch Weblinks, die unter ärztlicher Anleitung und Supervision eingesetzt werden sollten.

Eine etwas ausführlichere Darstellung von Synkopen als Differenzialdiagnose epileptischer Anfälle wäre bei dem Umfang des Gesamtwerkes wünschenswert gewesen. So fehlt beispielsweise das posturale Tachykardiesyndrom (POTS) junger Frauen vollständig. Auf die wichtige Differenzialdiagnose der kardialen konvulsiven Synkope wird zwar in einem Fallbeispiel (Seite 293) hingewiesen, aber beispielsweise das Romano-Ward-Syndrom hätte durchaus dezidiert dargestellt werden können. Trotz der kleinen Kritikpunkte ist dieses Buch nicht nur Neurologen und Neurologinnen, sondern allen zu empfehlen, die Patientinnen und Patienten mit Anfallsleiden betreuen. 